Electronic Supplemental Information (ESI)

\title{
Consumer-Grade Inkjet Printer for Versatile and Precise Chemical Deposition
}

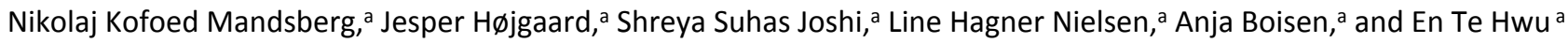

${ }^{a}$ Center for Intelligent Drug Delivery and Sensing Using Microcontainers and Nanomechanics (IDUN), Department of Health Technology,

Technical University of Denmark, 2800 Kgs. Lyngby, Denmark 


\section{Expanded section for Materials and Methods}

\section{Printer modifications}
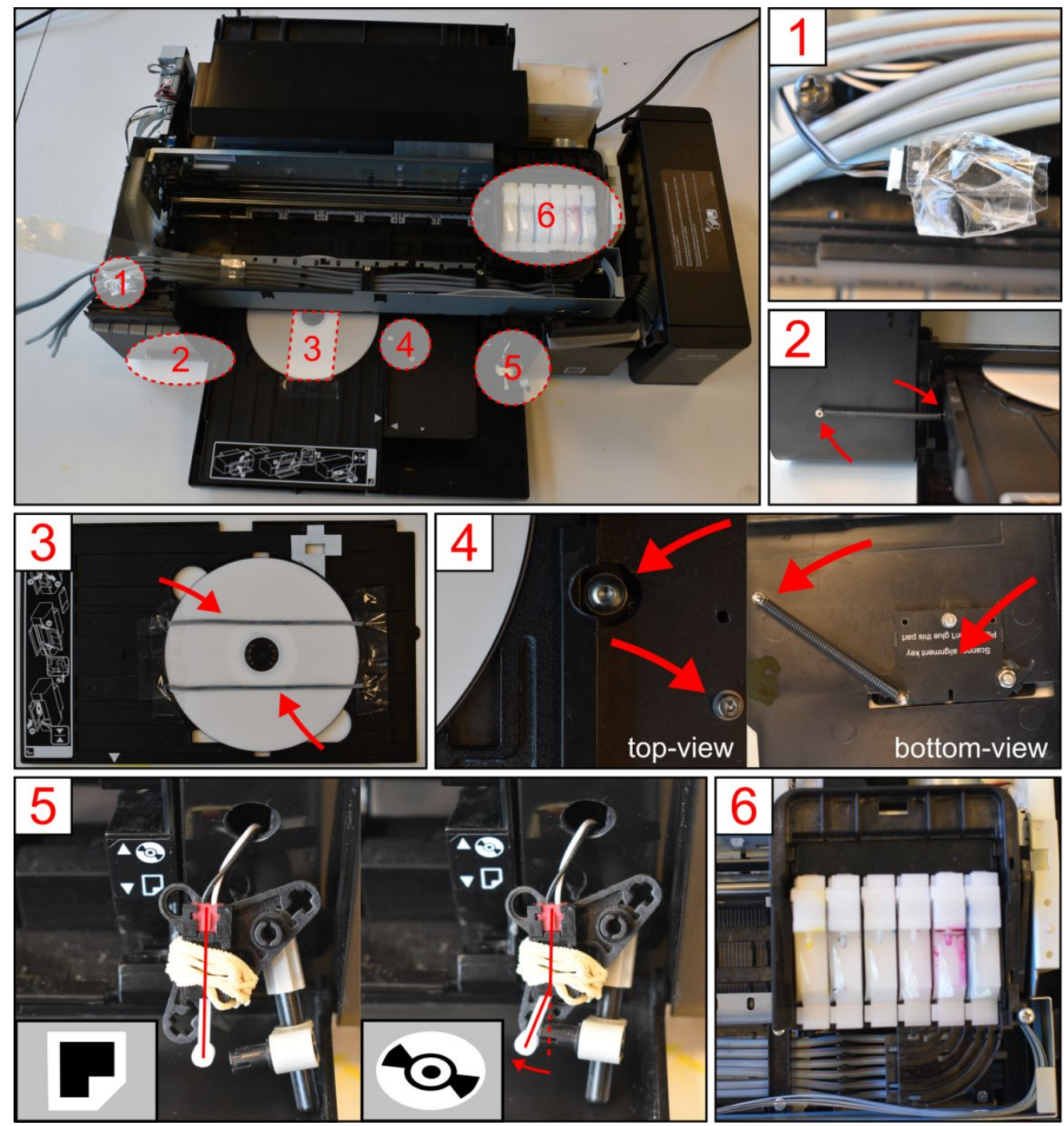

Figure S1 Overview of the modifications made to the inkjet printer. (top-left) The printer is shown in full with the different modifications numbered. (1) By-passing the lid-closure sensor by taping the mechanical switch. (2) Spring-loading of DVD tray holder to reduce wiggliness. (3) Roller isolation bars mounted on the DVD to enable printing on protruded substrates and multiple printing without smearing. (4) Spring-loading of DVD tray to close the gap between the DVD tray and its rail. (5) Extracted mechanical switch for tray position detection, thereby making reboot of the system possible without tray removal. (6) Ink cartridges with for alternative liquid print media.

Mostly for convenience, other modifications were made in addition to the two main ones. In this paragraph, we describe all of the modifications. Firstly, the lid closure sensor is a mechanical switch that can easily be taped to allow operation while the lid is open (Figure S1-1). The spring-loading presented in Figure S1-2 pulls the DVD tray holder toward the left to minimize wiggliness. In Figure S1-3, an example of roller isolation bars using electrical wires is shown and these enable printing on protruded substrates and multiple printing on the same substrate without smearing. In Figure S1-4, another spring-loading is shown that, via a ball-bearing, pushes the DVD tray against the left side of the DVD tray feeder to further reduce wiggle room and backlash induced movements. Figure S1-5, presents a LEGO switch for changing between the paper and DVD tray position. The switch is convenient as it allows rebooting the system without the need to remove and reinsert the DVD tray, which, otherwise, can easily cause misalignment. Finally, in Figure S1-6 we simply see the $\sim 2 \mathrm{~mL}$ cartridges in which the chosen chemicals are loaded. The cartridges not used in the specific demonstration were removed and their residues cleaned from the print head with $+2 \mathrm{~mL}$ acetone. 


\section{Homogenity analysis}
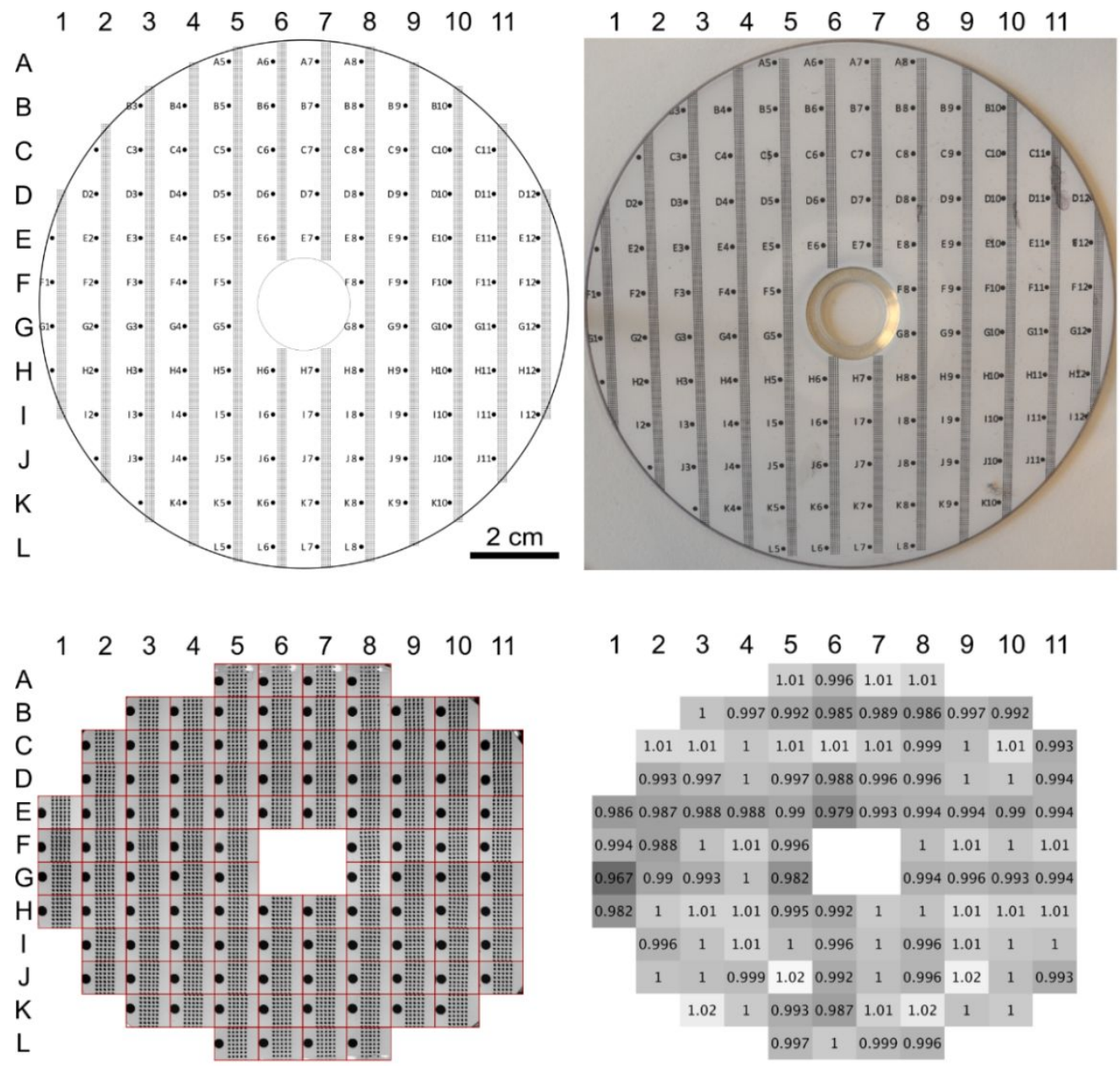
$\begin{array}{lllllllllll}1 & 2 & 3 & 4 & 5 & 6 & 7 & 8 & 9 & 10 & 11\end{array}$ $\begin{array}{llll}1.01 & 0.996 & 1.01 & 1.01\end{array}$

$\begin{array}{llll}1 & 0.9970 .9920 .9850 .9890 .9860 .9970 .992\end{array}$

\begin{tabular}{|cc|cccccccc}
1.01 & 1.01 & 1 & 1.01 & 1.01 & 1.01 & 0.999 & 1 & 1.01 & 0.993 \\
0.993 & 0.997 & 1 & 0.997 & 0.988 & 0.996 & 0.996 & 1 & 1 & 0.994
\end{tabular} $\begin{array}{lllllllllllllll}0.986 & 0.987 & 0.988 & 0.988 & 0.99 & 0.979 & 0.993 & 0.994 & 0.994 & 0.99 & 0.994\end{array}$ \begin{tabular}{lllll|lllll}
0.994 & 0.988 & 1 & 1.01 & 0.996 & 1 & 1.01 & 1 & 1.01
\end{tabular} $\begin{array}{lllllllllllllllllll}0.967 & 0.99 & 0.993 & 1 & 0.982 & & 0.994 & 0.996 & 0.993 & 0.994\end{array}$ $\begin{array}{lllllllllllll}0.982 & 1 & 1.01 & 1.01 & 0.995 & 0.992 & 1 & 1 & 1.01 & 1.01 & 1.01\end{array}$ $\begin{array}{lllllllllllll}0.996 & 1 & 1.01 & 1 & 0.996 & 1 & 0.996 & 1.01 & 1 & 1\end{array}$

$\begin{array}{lllllllllll}1 & 1 & 0.999 & 1.02 & 0.992 & 1 & 0.996 & 1.02 & 1 & 0.993\end{array}$ $\begin{array}{lllllllll}1.02 & 1 & 0.993 & 0.987 & 1.01 & 1.02 & 1 & 1\end{array}$ $\begin{array}{lll}0.997 \quad 1 & 0.999 & 0.996\end{array}$

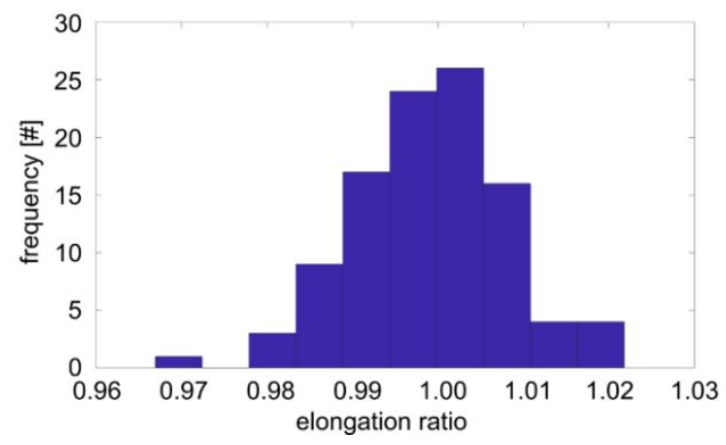

Figure S2 Pattern for testing print homogeneity across the DVD. (top-left) The pattern design consisting of $450 \mu \mathrm{m}$ squares spaced $450 \mu \mathrm{m}$ apart in a square pattern. Indices are present at the positions in the characterization. (top-right) Photograph of the pattern after printing on the DVD (middle-left) Microscopy images taken at indexed positions. (middle-right) For each position, the elongation of the square is calculated as the ratio between the average distance between centers of neighboring squares (determined as the peak location with the MATLAB findpeaks functions of a Gaussian smoothed image with std $=50$ pixels.) in orthogonal directions. (bottom) A histogram of the elongation ratios. The distribution is centered around 1, which indicates no elongation on average and thus the spread of the distribution is likely due to imperfections in the analysis or caused by the array direction of the nozzles in the print head. 
To test the parallelization of the method, we also characterized the homogeneity in the printing across the full DVD with respect to the shapes of submillimeter figures and potential elongation in those. The latter potentially being an issue of tray slipping that could challenge the ability of alignment. While no elongation was observed (Figure S2), the print showed some expected variation in the test shape. Unfortunately, since the expected print figures are further from the design shapes as compared to the. Dialog with the equipment manufacturer revealed that the difference may be related to the fact that the tray initially does not move during printing.

\section{Repeat analysis}

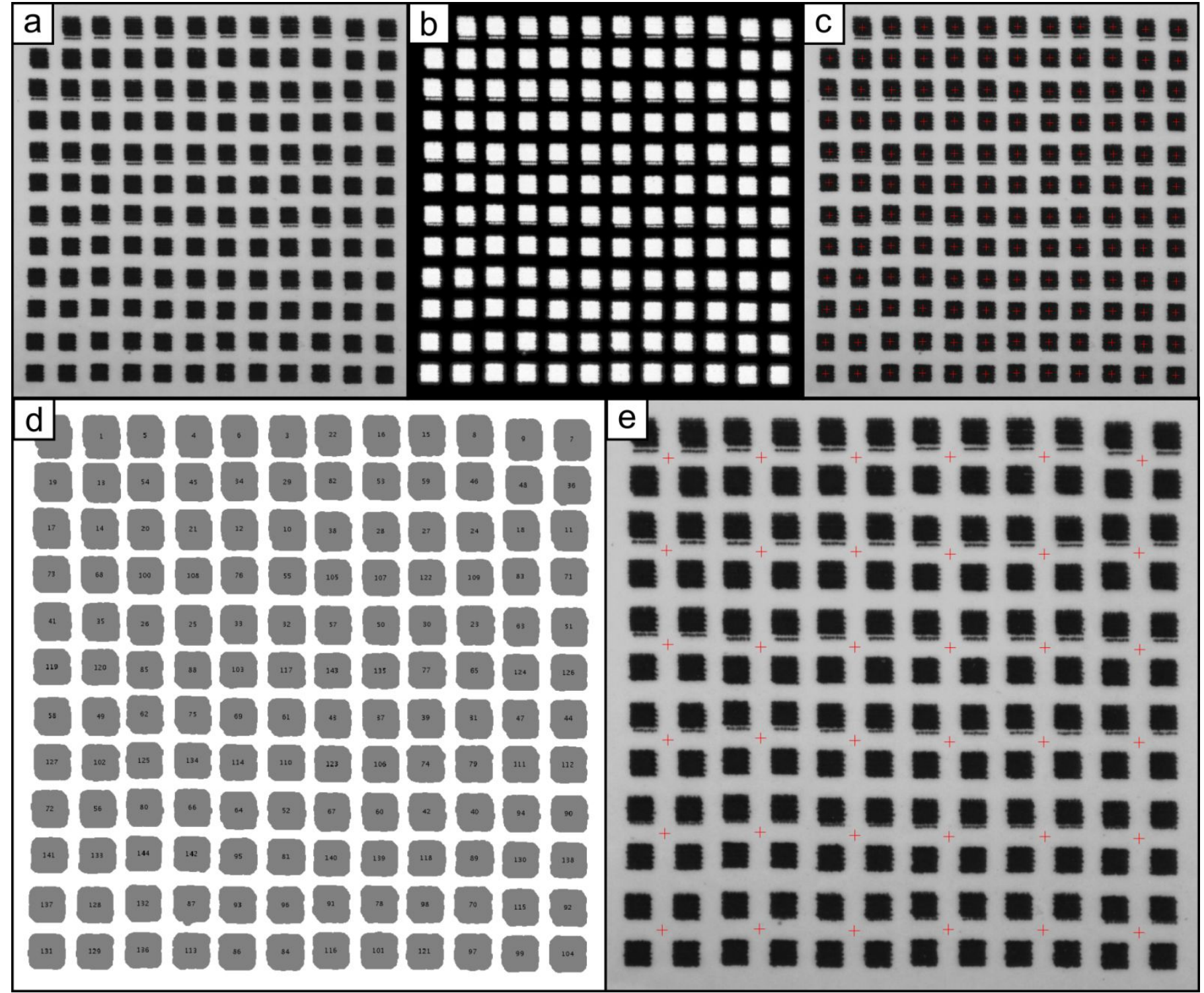

Figure S3 MATLAB analysis procedure for the characterization of printing repeatability. (a) The optical microscopy image of the unit cell ( 2 by 2 squares with both side length and spacing of $450 \mu \mathrm{m}$ ) stepped in a 6 by 6 array with a step-size of $1800 \mu \mathrm{m}$. (b) The image is inverted and background corrected. (c) The individual squares are found using the connected component function and the centers are calculated. (d) A mask containing all the individual squares and a small surrounding region is defined. (e) The centers of the printed unit cells are calculated using an average position of the pixels contained in the masked region weighted by the background corrected pixel intensities. 


\section{Demonstrations}

\section{Drug loading}
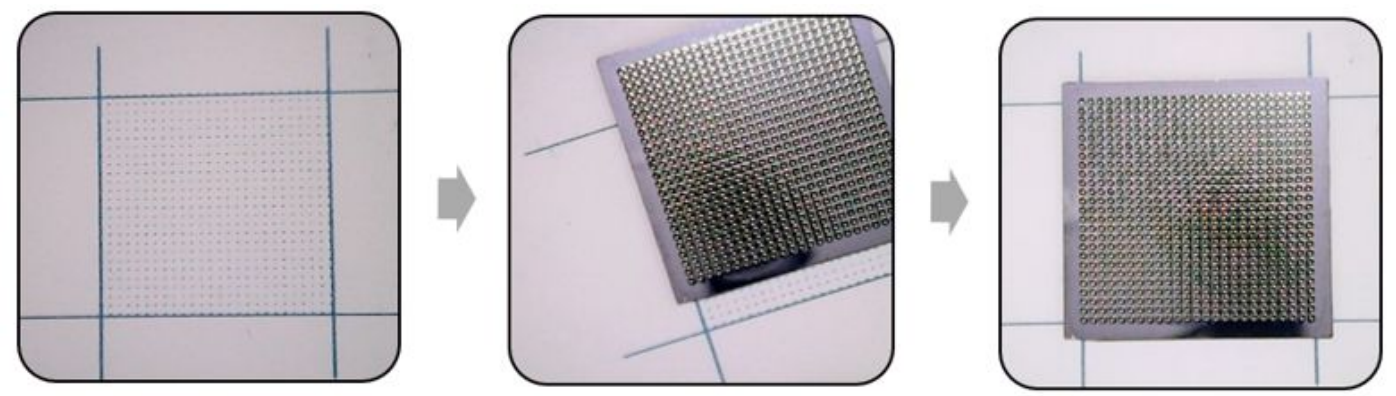

Figure S4 Alignment method for chip with microcontainers. (left) The alignment pattern initially printed on the DVD. (middle) The chip is placed on the alignment pattern and (right) its position is adjusted with a micromanipulator under a microscope.

\section{Microfluidics}
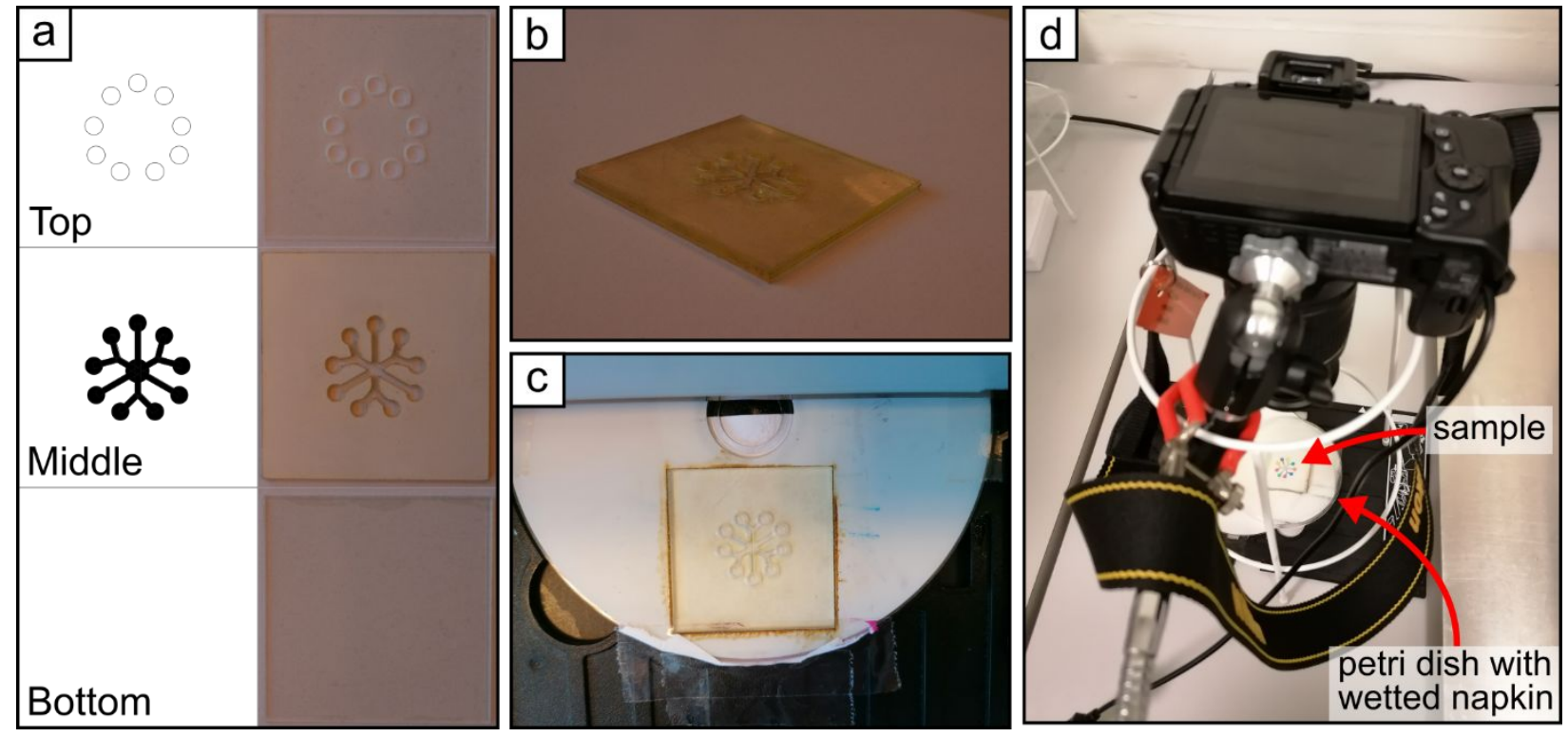

Figure S5 Layer-by-layer assembly of microfluidic system and diffusion test setup. (a) The three layers of the microfluidic system is shown with the design on the left and the realization on the right. (b) Tilted photograph of the assembled microfluidic chip. (c) With a $\mathrm{CO}_{2}$-laser, a DVD was cut to act as a mount for the chip. (d) Following the quasi-simultaneous printing, the chip was covered by a Petri dish with wet napkins inside to reduce evaporation speed by saturating the atmosphere with water vapor. A time-series of photographs were captured with a Nikon D5600 DSLR camera for $36 \mathrm{hr}$ during the diffusive mixing experiment.

\section{Bacteria studies}

\section{Bacteria Studies - Gradient Assays with Anti-Biotic for Combinatorial Minimum Inhibition Concentrations}

Knowledge about bacterial resistance toward and inhibition by anti-biotic is crucial for safe use. Therefore, a standard experiment is to incubate the bacteria of interest with the anti-biotic subject to investigation. This is done at multiple concentrations of the antibiotic and it is observed how levels above a certain threshold (the minimum inhibition concentration) suppresses cell multiplication. The experiment is performed in multiple vials, making chemical consumption large. By using inkjet gradient printing, the chemical consumption could be significantly reduced and likewise could the physical size of the experiment. Often doctors prescribe combinations of antibiotic. When interested in these combinatorial responses, the number of experiments required increases dramatically. In such cases, the creation of miniaturized gradient arrays is extra important, as the experimental footprint would otherwise be unfeasibly large. We made an agar plate inset for the printer that can be used for such studies. The agar plates, tailored to fit the inkjet printer DVD tray, were fabricated from two pieces of laser cut PMMA sheets (100 
$\mu \mathrm{m}$ thick) bonded together with double-sided tape (see Figure S6a-(i) for the layout). The plates were then illuminated with UV for two minutes (400 W, ECE 5000 UV Light-Curing Flood Lamp, Dymax, USA). To make the agar thickness compatible with the printer dimensions, the plates had their wettability increased through a two-minute low-pressure oxygen plasma exposure (Atto Plasma Cleaner, Diener Electronic) with conditions: $50 \mathrm{~W}, 40 \mathrm{~Pa} .{ }^{\mathrm{S} 1, \mathrm{~S} 2}$ Within 5 minutes, LG growth agar was pipetted into the $100-\mu \mathrm{m}$ deep wells and let to solidify before a known volume of bacteria stock can be spread across it with an inoculation loop. The plate with agar (Figure S6a-(ii)) and bacteria is mounted on the DVD tray together with the roller isolators (Figure S6a-(iii)) and spatially orthogonal $1 \mathrm{D}$ gradient patterns of $1 \mathrm{mg} / \mathrm{mL}$ amoxicillin trihydrate (with $\sim 5 \mathrm{vol} \%$ Epson cyan ink) and $10 \mathrm{mg} / \mathrm{mL}$ ciprofloxacini hydrochloridium (with $5 \mathrm{vol} \%$ red food dye) dissolved in $18.2 \mathrm{M} \Omega$ Milli-Q were printed (Figure S6b-(ii) although the ciprofloxacin cannot be visualized due to the small concentration of the dye).

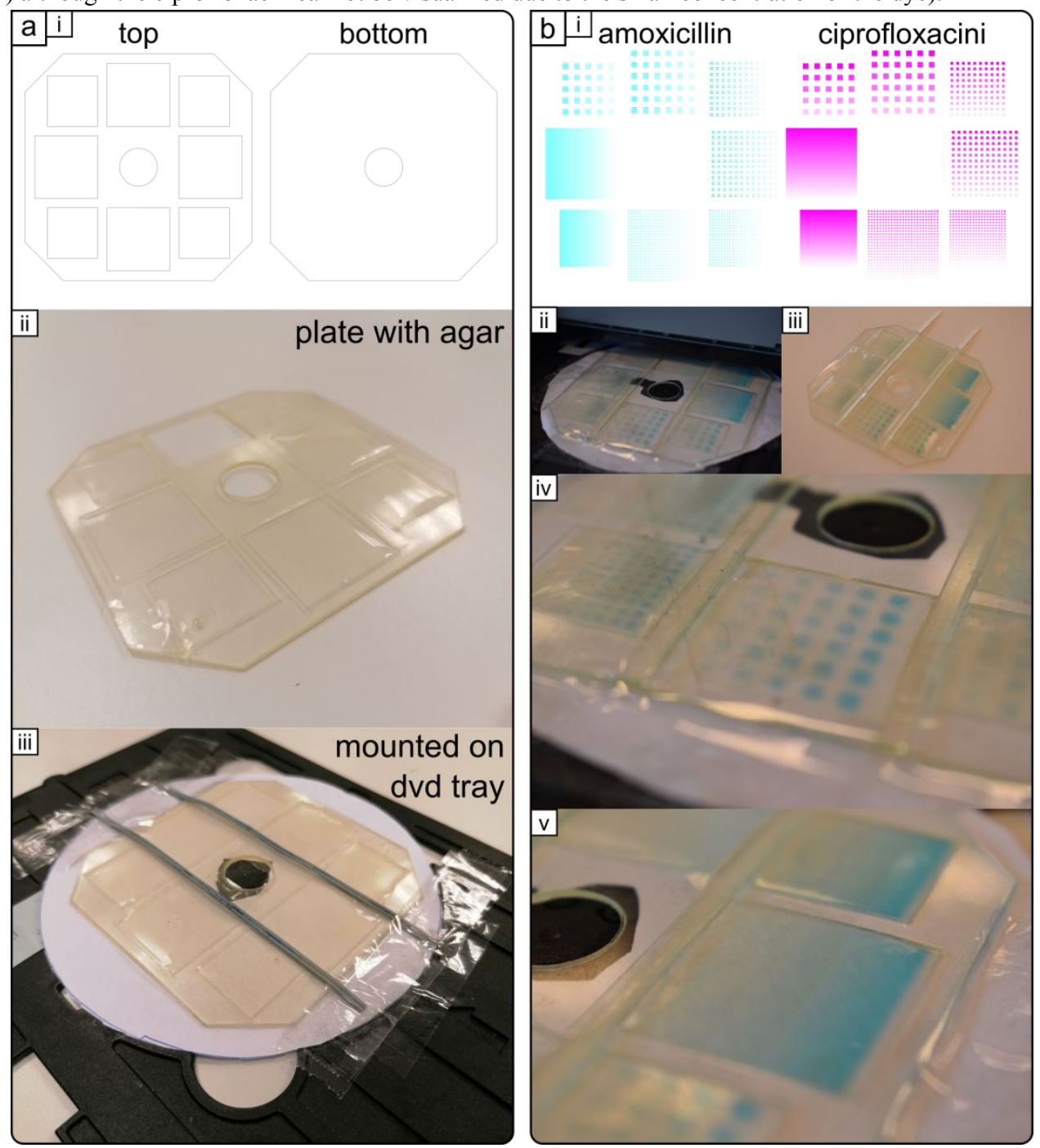

Figure S6 Agar plate inset for the inkjet printer and combinatorial gradient array. (a-i) Top and bottom part layout of the agar plate printer inset. (a-ii) Photograph of the assembled agar plate with agar pipette in the wells. (a-iii) The agar plate inset mounted on the DVD tray. (b-i) The two mutually orthogonal gradient patterns for combinatorial studies of minimum inhibition concentration of antibiotics. (b-ii/iii) The printed gradients on the full agar plate. (b-iv/v) Close-up of two gradient fields; one with discrete regions of antibiotic and one with a continuous gradient hereof.

\section{References}

(S1) Schneider, L.; Laustsen, M.; Mandsberg, N.; Taboryski, R. The Influence of Structure Heights and Opening Angles of Micro- and Nanocones on the Macroscopic Surface Wetting Properties. Sci. Rep. 2016, 6 (February), 21400. https://doi.org/10.1038/srep21400. Telecka, A.; Mandsberg, N. K.; Li, T.; Ludvigsen, E.; Ndoni, S.; Di Mundo, R.; Palumbo, F.; Fiutowski, J.; Chiriaev, S.; Taboryski, R. Mapping the Transition to Superwetting State for Nanotextured Surfaces Templated from Block-Copolymer Self-Assembly. Nanoscale 2018, 10 (44), 20652-20663. https://doi.org/10.1039/c8nr07941b. 